\title{
Note A Matrix Trace Inequality
}

\author{
Feng Zhang*, Lin Deng, Jinli Xu \\ Department of mathematics, Northeast Forestry University, Harbin, China, 150040
}

*Corresponding Author: Feng Zhang, Department of mathematics, Northeast Forestry University, Harbin, China, 150040

Abstract: In this note, we generalized the matrix trace inequality which is given by Yang, and the following result $\left|\operatorname{tr}\left(A_{1} A_{2} \ldots A_{k}\right)^{m}\right| \leq\left[\operatorname{tr}\left(A_{1}^{k m}\right) \operatorname{tr}\left(A_{2}^{k m}\right) \operatorname{tr}\left(A_{k}^{k m}\right)\right]^{\frac{1}{k}}$ is established, where $m, k$ are positive integers, $A_{1}, \ldots, A_{k}$ are positive semidefinite matrix of the same order.

Keywords: trace inequality positive semidefinite matrix

\section{INTRODUCTION}

In paper [1],yang improves Bellmann's result and get the trace inequalities: For $n=1,2, \ldots$

$0 \leq \operatorname{tr}(A B)^{2 n} \leq(\operatorname{tr} A)^{2}\left(\operatorname{tr} A^{2}\right)^{n-1}\left(\operatorname{tr} B^{2}\right)^{n}$

$0 \leq \operatorname{tr}(A B)^{2 n+1} \leq(\operatorname{tr} A)(\operatorname{tr} B)\left(\operatorname{tr} A^{2}\right)^{n}\left(\operatorname{tr} B^{2}\right)^{n}$

$A, B$ are positive semidefinite matrices of the same order.

Next, yang[4] improves this inequality: $A, B$ are positive semidefinite matrices of the same order, then for any positive integer $m, \operatorname{tr}(A B)^{2 m} \leq\left\{\operatorname{tr}(A)^{2 m} \operatorname{tr}(B)^{2 m}\right\}^{\frac{1}{2}}$. The purpose of this paper is to generalized the above matrix trace inequalites. we start with the following lemmas:

The vector of eigenvalues of $A$ is denoted by $\lambda(A)=\left(\lambda_{1}(A), \lambda_{2}(A), \ldots, \lambda_{n}(A)\right)$ If $A$ is Hermitian , we arrange the eigenvalues of $A$ in nonincreasing order, $\lambda_{1}(A) \geq \lambda_{2}(A) \geq \ldots \geq \lambda_{n}(A)$.In addition $x \prec y$ means that $x=\left(x_{1}, x_{2}, \ldots, x_{n}\right)$ is majorized by $y=\left(y_{1}, y_{2}, \ldots, y_{n}\right)$ with $x_{1} \geq x_{2} \geq \ldots \geq x_{n}$ and $y_{1} \geq y_{2} \geq \ldots \geq y_{n}$, if we have $\sum_{i=1}^{k} x_{i} \leq \sum_{i=1}^{k} y_{i}(k=1, \ldots, n-1)$ and $\sum_{i=1}^{n} x_{i}=\sum_{i=1}^{n} y_{i} . x \prec_{w} y$ means that $x=\left(x_{1}, x_{2}, \ldots, x_{n}\right)$ is weak majorized by $y=\left(y_{1}, y_{2}, \ldots, y_{n}\right)$, if we have $\sum_{i=1}^{k} x_{i} \leq \sum_{i=1}^{k} y_{i}(k=1, \ldots, n)$.

Lemma1[3] Let $a_{i j}(i=1, \ldots, n ; j=1, \ldots, m)$ be nonnegative real numbers, and $b_{1}, \ldots, b_{m}$ are positive 
integers with $\frac{1}{b_{1}}+\ldots+\frac{1}{b_{m}}=1$. Then $\sum_{i=1}^{n} a_{i 1} \ldots a_{i m} \leq\left(\sum_{i=1}^{n} a_{i 1}^{b_{1}}\right)^{\frac{1}{b_{1}}} \ldots\left(\sum_{i=1}^{n} a_{i m}^{b_{m}}\right)^{\frac{1}{b_{m}}}$.

Lemma2[6] $A_{1}, \ldots, A_{m}$ are $n$-square complex matrices, then $s\left(\prod_{j=1}^{m} A_{j}\right) \prec_{w}\left\{\prod_{j=1}^{m} s_{i}\left(A_{j}\right)\right\}_{i=1}^{n}$.

Lemma3[3] Suppose $f(t)$ is a monotonically increasing function, then $x \prec_{w} y$ contains $\left(f\left(x_{1}\right), \ldots, f\left(x_{n}\right)\right) \prec_{w}\left(f\left(y_{1}\right), \ldots, f\left(y_{n}\right)\right)$.

Lemma4[5] Denoted the eigenvalues of matrix $A$ by $\lambda(A)=\left(\lambda_{1}, \ldots, \lambda_{n}\right)$, then $\left\{\left|\lambda_{i}\right|\right\}_{i=1}^{n} \prec_{w} s(A)$. Specially,$|\operatorname{tr} A| \leq \sum_{i=1}^{n} s_{i}(A)$.

2 Main results

Theorem5 $A_{1}, \ldots, A_{k}$ are positive semidefinite matrix of order $\mathrm{n}$, then for positive integer $m, k$ $\left|\operatorname{tr}\left(A_{1} A_{2} \ldots A_{k}\right)^{m}\right| \leq\left[\operatorname{tr}\left(A_{1}^{k m}\right) \operatorname{tr}\left(A_{2}^{k m}\right) \operatorname{tr}\left(A_{k}^{k m}\right)\right]^{\frac{1}{k}}$

Proof According to Lemma4, it's obvious that $\left|\operatorname{tr}\left(A_{1} A_{2} \ldots A_{k}\right)^{m}\right|=\left|\sum_{i=1}^{n} \lambda_{i}\left(A_{1} A_{2} \ldots A_{k}\right)^{m}\right| \leq \sum_{i=1}^{n}\left|\lambda_{i}\left(A_{1} A_{2} \ldots A_{k}\right)^{m}\right| \leq \sum_{i=1}^{n} s_{i}\left(A_{1} A_{2} \ldots A_{k}\right)^{m}$

Next we use Lemma1-3 can prove $\left|\operatorname{tr}\left(A_{1} A_{2} \ldots A_{k}\right)^{m}\right| \leq \sum_{i=1}^{n} s_{i}^{m}\left(A_{1}\right) s_{i}^{m}\left(A_{2}\right) \cdots s_{i}^{m}\left(A_{k}\right)$

and

$\sum_{i=1}^{n} s_{i}^{m}\left(A_{1}\right) s_{i}^{m}\left(A_{2}\right) \cdots s_{i}^{m}\left(A_{k}\right) \leq\left\{\sum_{i=1}^{n} s_{i}^{k m}\left(A_{1}\right) \sum_{i=1}^{n} s_{i}^{k m}\left(A_{2}\right) \cdots \sum_{i=1}^{n} s_{i}^{k m}\left(A_{k}\right)\right\}^{\frac{1}{k}}$

On the other hand, we notice $s_{i}^{k m}\left(A_{i}\right)=\lambda_{i}\left(A_{i}^{k m}\right), \quad(1 \leq i \leq n)$. Therefore $\left\{\sum_{i=1}^{n} s_{i}^{k m}\left(A_{1}\right) \sum_{i=1}^{n} s_{i}^{k m}\left(A_{2}\right) \cdots \sum_{i=1}^{n} s_{i}^{k m}\left(A_{k}\right)\right\}^{\frac{1}{k}}=\left[\operatorname{tr}\left(A_{1}\right)^{k m} \operatorname{tr}\left(A_{2}\right)^{k m} \operatorname{tr}\left(A_{k}\right)^{k m}\right]^{\frac{1}{k}}$.

So we can get $\left|\operatorname{tr}\left(A_{1} A_{2} \ldots A_{k}\right)^{m}\right| \leq\left[\operatorname{tr}\left(A_{1}^{k m}\right) \operatorname{tr}\left(A_{2}^{k m}\right) \operatorname{tr}\left(A_{k}^{k m}\right)\right]^{\frac{1}{k}}$

Corollary $6^{[4]} A, B$ are positive semidefinite matrices of the same order, then for positive integer $m$, $\operatorname{tr}(A B)^{m} \leq\left[\operatorname{tr}(A)^{2 m} \operatorname{tr}(B)^{2 m}\right]^{\frac{1}{2}}$

Corollary $7^{[1]} A, B$ are positive semidefinite matrices of the same order, then for $n=1,2, \ldots$

$0 \leq \operatorname{tr}(A B)^{2 n} \leq(\operatorname{tr} A)^{2}\left(\operatorname{tr} A^{2}\right)^{n-1}\left(\operatorname{tr} B^{2}\right)^{n}$ 
$0 \leq \operatorname{tr}(A B)^{2 n+1} \leq(\operatorname{tr} A)(\operatorname{tr} B)\left(\operatorname{tr} A^{2}\right)^{n}\left(\operatorname{tr} B^{2}\right)^{n}$

Corollary $8 A_{1}, \ldots A_{k}$ are positive semidefinite matrix of order $\mathrm{n}$, then for positive integer $m, k$

$\left|\operatorname{tr}\left(A_{1} A_{2} \ldots A_{k}\right)^{m}\right| \leq \operatorname{tr}\left(A_{1}^{m}\right) \operatorname{tr}\left(A_{2}{ }^{m}\right) \operatorname{tr}\left(A_{k}{ }^{m}\right)$

\section{REFERENCES}

[1] X. Yang,A matrix trace inequality[J]. Math. Anal. Appl, 250 (2000): 372-374

[2] R.A. Horn, C.R. Johnson,Topics in Matrix Analysis, Cambridge Univ.Press, Cambridge (1991)

[3] D.S. Mitrinovic,Analytic Inequalities, Springer-Verlag, New York (1970)

[4] X. Yang,A matrix trace inequality[J]. Math. Anal. Appl, 263(2001): 327-331

[5] A.Horn, On the singular values of a product of completely continuous operators, Proc, National Acad. Sciences (U.S.)36 (1950), 374-375

[6] A.Horn, On the eigenvalues of a matrix with prescribed singular values, Proc. Amer. Math. Soc., $5(1954), 4-7$

Citation: Feng Zhang, et.al., (2019). Note A Matrix Trace Inequality. International Journal of Scientific and Innovative Mathematical Research (IJSIMR), 7(7), pp. 12-14. http://dx.doi.org/ 10.20431/2347 $-3142.0707004$

Copyright: () 2019 Authors, this is an open-access article distributed under the terms of the Creative Commons Attribution License, which permits unrestricted use, distribution, and reproduction in any medium, provided the original author and source are credited. 